\title{
Angiotensin Converting Enzyme-2 (ACE2) Receptors, asthma and severe COVID-19 infection risk
}

${ }^{1}$ Division of Allergy and Immunology, Department of Pulmonary Medicine, School of Medicine, Koç University, Istanbul, Turkey

${ }^{2}$ Department of Pulmonary Medicine, School of Medicine, Koç University, Istanbul, Turkey

\section{KEY wORDS}

Asthma; severe asthma; infection; COVID-19;

ACE2; TMPRSS2; SARS-CoV-2.

\author{
Corresponding author \\ Ayse Bilge Ozturk \\ Division of Allergy and Immunology \\ Department of Pulmonary Medicine \\ School of Medicine \\ Koç University Hospital \\ Davutpasa Caddesi 4 \\ 34010 Topkapi \\ Istanbul, Turkey \\ E-mail: aysebilgeozturk@yahoo.com
}

Doi

10.23822/EurAnnACI.1764-1489.169

\section{Abbreviations}

ACE2: Angiotensin converting enzyme-2

BAL: Bronchoalveolar lavage

CDC: Centers for Disease Control and Prevention

COVID-19: Coronavirus disease
COPD: Chronic obstructive pulmonary disease

HBECs: Bronchial epithelial cells

IL: Interleukin

SARS-CoV-2: SARS-coronavirus 2

TMPRSS2: transmembrane protease serine 2

\section{Highlights}

- SARS-CoV-2 uses the ACE2 receptors for host cell entry.

- ACE2 receptor gene expression in airways seems to be similar in asthma and health.

- Asthma does not seem to be a risk factor for severe COVID-19.
- T2 high inflammation, inhaler steroid use may have an impact on ACE receptor gene downregulation.

- ACE2 receptor gene expression may vary in central and peripheral airways.

- ACE2 receptor gene expression may differ in various asthma endotypes and some subgroups such as smokers may have more risk for COVID-19. 
To Editor,

Coronavirus disease 2019 (COVID-19), was first detected in Wuhan, China, and it has since spread to most countries around the world. A recent metanalysis including studies from China have shown that preexisting chronic disease including hypertension, diabetes, cardiovascular and respiratory system diseases is one of the main risk factors for mortality in adult COVID-19 inpatients (1). When severe and non-severe COVID-19 patients are compared, higher risk of respiratory system disease with the odds ratio of 2.46 has been detected (1).

Asthma is one of the most common chronic respiratory diseases in the world and it is possible that asthma-related factors may influence susceptibility to COVID-19 or infection severity. So, there is a great concern about the effect of asthma on COVID-19 morbidity: Centers for Disease Control and Prevention (CDC) stated that people with moderate to severe asthma may have an increased risk for COVID-19 and infection may lead to an asthma attack, pneumonia, or acute respiratory disease. On the other hand, ARIA-EAACI statement on Asthma and COVID-19, reported that asthma does not seem to be a risk factor for severe COVID-19 (2). Because surprisingly a low rate of asthma has been reported in patients having COVID-19 (3,4). In Zhang et al. study including $140 \mathrm{com}-$ munity-infected COVID-19 patients, asthma or other allergic diseases were not reported by any of the patients (3). Although the prevalence of asthma in China was $4.2 \%$ and allergic rhinitis in Wuhan was 9.7\%, allergy or asthma was not detected as a risk factor for COVID-19 infection (3). In another article from Wuhan, Li et al. reported the prevalence of asthma as $0.9 \%$ in 548 patients with COVID-19 and the asthma rate did not differ between severe and non-severe COVID-19 cases (4). Available data is limited, there is no concrete evidence that asthma is a risk factor for COVID-19 and it needs further investigation whether asthma itself or the treatments used in asthma modification have a protective or causal effect on COVID-19 severity.

\section{The link between ACE receptors and COVID-19}

Angiotensin Converting Enzyme-2 (ACE2) receptors mediate the entry of SARS-coronavirus 2 (SARS-CoV-2) into target cells via its structural spike glycoprotein $(S)$, and the spike protein of SARS-CoV-2 is primed by transmembrane protease serine 2 (TMPRSS2) which allows fusion of viral and cellular membranes into host cells (5). ACE2 receptors are expressed in the heart, vessels, gut, lung, kidney, testis, and brain (6). Binding of SARS-CoV-2 to ACE2 receptors markedly down-regulates ACE2 receptors which have protective biological effects on human body (6). With the loss of the protective effect of these receptors interstitial fibrosis, endothelial dysfunction, enhanced inflammation, oxidative stress and increased coagulation can be seen (6). It is interesting to note that severity of the COVID-19 disease is associated with several conditions which may have ACE2 deficiency such as older age, male gender, hypertension, diabetes, or cardiovascular disease.

\section{Complex interplay between asthma, ACE2/TMPRSS2 receptors, Th2 high inflammation, inhaler corticosteroids and COVID-19}

ACE2 deficiency and its association with asthma is not clear yet. Peters et al. investigated differences in ACE2 and TMPRSS2 gene expression in sputum cells of 330 asthma patients and 79 healthy controls (7). Gene expression of ACE2 and TMPRSS2 was found similar in asthmatics and healthy controls. Among asthma patients, higher expressions of ACE2 and TMPRSS2 were observed in males, African Americans, and patients with diabetes mellitus. Interestingly, use of inhaled corticosteroids was associated with lower expression of ACE2 and TMPRSS2 after adjustment for asthma severity.

This is an important study giving clues about possible factors explaining the low prevalence of asthma among COVID-19 patients. Asthma itself or the use of inhaled steroids may have protective effect against SARS-CoV-2 infection. However, there are some limitations. ACE2 receptors are particularly expressed in type 2 pneumocytes which have an effective role on triggering a cascade of inflammation in the lower respiratory tract. Sputum may not reflect the amount of ACE2 receptors in the lower respiratory tract including type 2 pneumocytes. Many of the inhaler steroids have less peripheral airway deposition and so inhaler steroids might not effect ACE2 expression of type 2 pneumocytes. Smoking status of the patients and asthma endotypes (eosinophilic or neutrophilic, atopic, non-atopic) were not provided in Peters et al. study.

Radzikowska et al. analyzed ACE2 and TMPRSS2 gene expression in primary Human Bronchial Epithelial Cells (HBECs), bronchial biopsies and bronchoalveolar lavage fluid of healthy children/adults and adult asthma and COPD patients (8). They did not see any significant difference in ACE2 expression in HBECs or bronchial biopsy between control, asthma and COPD patients. However, ACE2 expression in bronchial biopsies found to be higher in smokers. TMPRSS2 expression were high in HBECs of asthmatic patients. These results suggest that smoking status have an enhancing effect on ACE2 expression which may have a positive impact on the entry of SARS-CoV-2 into lung cells and negative impact on COVID-19 severity. Even though TMPRSS2 was found similar in asthmatics and healthy controls in Peters et al. study, Radzikowska et al. observed higher expression of TMPRSS2 in asthmatic airways. The difference between two study may be related with the use of different samples for the analysis of 
Table I - ACE2 and TMPRSS2 expression in nasal epithelial cells, sputum and bronchial biopsy, and human bronchial epithelial cells in asthma.

\begin{tabular}{|c|c|c|c|c|c|c|c|c|c|c|c|c|c|c|c|c|c|c|c|c|c|c|c|c|}
\hline & \multicolumn{5}{|c|}{ Nasal Epithelial Cells } & \multicolumn{7}{|c|}{ Sputum } & \multicolumn{6}{|c|}{ Bronchial Biopsy } & \multicolumn{6}{|c|}{ HBEC } \\
\hline Peters et al ${ }^{8}$ & NA & NA & NA & NA & NA & NA & $\leftrightarrow$ & NA & NA & $\leftrightarrow$ & NA & NA & NA & NA & NA & NA & NA & NA & NA & NA & NA & NA & NA & NA \\
\hline
\end{tabular}

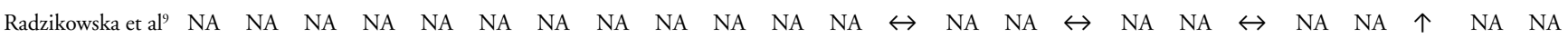

$\begin{array}{lllllllllllllllllllllllllllllll}\text { Sajuthi et } \mathrm{a}^{10} & \downarrow & \text { NA } & \text { NA } & \uparrow & \text { NA } & \text { NA } & \text { NA } & \text { NA } & \text { NA } & \text { NA } & \text { NA } & \text { NA } & \text { NA } & \text { NA } & \text { NA } & \text { NA } & \text { NA } & \text { NA } & \text { NA } & \text { NA } & \text { NA } & \text { NA } & \text { NA } & \text { NA }\end{array}$

$\downarrow=$ decreased; $\uparrow=$ increased; $\leftrightarrow$ = no significant difference; NA = not analysed; ACE2:Angiotensin converting enzyme-2; BAL: Bronchoalveolar lavage; HBECs: Bronchial epithelial cells: TMPRS2: Transmembrane protease serine 2.

ACE2 expression: sputum in Peters et al. study versus bronchial biopsy in Radzikowska et al. study. It might also be related to the heterogeneity of asthma endotypes such as eosinophilic (Th2-high) asthma which is most likely seen in atopics or neutrophilic (Th2-low) asthma which is most likely seen in smokers.

Asymptomatic nasal carriage of COVID-19 is more common in children and children mostly seems not to have a severe COVID-19 infection. Therefore, to understand the association between ACE receptors and childhood asthma which is mainly eosinophilic or Th2 high endotype may help to understand the possible link between Th2 high asthma endotype and COVID-19 infection severity. Sajuthi et al. used nasal airway transcriptome and network co-expression analysis to identify the cellular and transcriptional factors in COVID-19 infectivity (9). They used a children cohort including 695 subjects with asthma and healthy controls between the ages of 8 and 21. They mainly focused on ACE2 and TMPRSS2 expression. They found that interleukin (IL)-13 mediated Th2 high inflammation had a major role in ACE2 downregulation and TMPRSS2 upregulation. Th2-low and Interferon-high individuals were found to express high level of ACE2. They also showed that ACE2 was expressed in secretory cells and ciliated cells while TMPRSS2 was expressed by all epithelial cell types (9). The results of this study can be interpreted as Th2 rich inflammation may have protective role against COVID-19 by causing ACE2 downregulation. Virus behavior may be different depending on ACE2 and TMPRSS2 expression variations in different part of the airways such as nasal and peripheral airways. TMPRSS2 may have more effective role in nose compared to ACE2. ACE2 and TMPRSS2 expression in nasal epithelial cells, sputum and bronchial biopsy, and human bronchial epithelial cells in asthma is summarized in table $\mathbf{I}$.

In conclusion, it is not yet to be proved that asthma is a risk factor for COVID-19 infection. Whether there is a link between asthma and COVID-19 infection remains to be determined. The heterogeneous nature of asthma may cause interindividual variation in COVID-19 infection immunology. More clinical studies focusing ACE2 and TMPRSS2 expression in central and peripheral airways are warranted to understand the role of individual factors such as atopy, obesity and smoking habit and treatment related factors such as inhaled/systemic steroid use in different asthma groups (mild/severe, Th2-high and Th2-low asthma) for the risk of COVID-19 morbidity.

\section{Conflict of interests}

The authors declare that they have no conflict of interests.

\section{References}

1. Yang J, Zheng Y, Gou X, et al. Prevalence of comorbidities in the novel Wuhan coronavirus (COVID-19) infection: a systematic review and meta-analysis. Int J Infect Dis 2020;94:91-95.

2. Bousquet J, Jutel M, Akdis CA, et al. ARIA-EAACI statement on asthma and COVID-19 (June 2, 2020). Allergy 2020:10.1111/ all.14471. 
3. Zhang JJ, Dong X, Cao YY, et al. Clinical characteristics of 140 patients infected with SARS-CoV-2 in Wuhan, China. Allergy 2020;75(7):1730-1741.

4. Li X, Xu S, Yu M, et al. Risk factors for severity and mortality in adult COVID-19 inpatients in Wuhan. J Allergy Clin Immunol 2020;146(1):110-118.

5. Hoffmann M, Kleine-Weber H, Schroeder S, et al. SARS-CoV-2 Cell Entry Depends on ACE2 and TMPRSS2 and Is Blocked by a Clinically Proven Protease Inhibitor. Cell 2020;181(2):271-280.e8.

6. Verdecchia P, Cavallini C, Spanevello A, Angeli F. The pivotal link between ACE2 deficiency and SARS-CoV-2 infection. Eur J Intern Med 2020;76:14-20.
7. Peters MC, Sajuthi S, Deford P, et al. COVID-19 Related Genes in Sputum Cells in Asthma: Relationship to Demographic Features and Corticosteroids. Am J Respir Crit Care Med 2020;202(1):83-90.

8. Radzikowska U, Ding M, Tan G, et al. Distribution of ACE2, CD147, CD26, and other SARS-CoV-2 associated molecules in tissues and immune cells in health and in asthma, COPD, obesity, hypertension, and COVID-19 risk factors. Allergy 2020:10.1111/ all.14429.

9. Sajuthi SP, DeFord P, Jackson ND, et al. Type 2 and interferon inflammation strongly regulate SARS-CoV-2 related gene expression in the airway epithelium. bioRxiv [Preprint]. 2020:2020.04.09.034454. 